\title{
Platinum-based Agent and Fluorouracil in Metastatic Breast Cancer: A Retrospective Monocentric Study with a Review of the Literature
}

\author{
LORENZO ROSSI ${ }^{1,2,3}$, CHIARA BIAGIONI ${ }^{1}$, AMELIA MCCARTNEY ${ }^{1}$, ERICA MORETTI ${ }^{1}$, MARTA PESTRIN ${ }^{1}$, \\ GIUSEPPINA SANNA ${ }^{1}$, EMANUELA RISI ${ }^{1}$, LUCA MALORNI ${ }^{1}$, ANGELO DI LEO ${ }^{1}$ and LAURA BIGANZOLI ${ }^{1}$ \\ ${ }^{1}$ Sandro Pitigliani Medical Oncology Department, Hospital of Prato, Prato, Italy; \\ ${ }^{2}$ Institute of Oncology of Southern Switzerland (IOSI), Bellinzona, Switzerland; \\ ${ }^{3}$ Breast Unit of Southern Switzerland (CSSI), Lugano, Switzerland
}

\begin{abstract}
Background: The combination of platinum with 5-fluorouracil has scarcely been studied in metastatic breast cancer. As this combination does not lead to significant hepatic metabolism, in some clinical situations it may prove useful, especially in cases with liver dysfunction and an urgent clinical need for rapid tumor shrinkage. A retrospective study was conducted to evaluate the efficacy and safety of the combination of cisplatin and 5-fluorouracil in patients with metastatic breast cancer with significant alterations of biochemistry. Patients and Methods: A total of 109 patients with metastatic breast cancer and liver dysfunction were treated; time-to-progression, overall survival and trends in liver function were evaluated. Results: The median time-to-progression was 3.4 months, and median overall survival was 7.8 months. About 50\% of patients obtained a complete, partial or stable biochemical response and 24 patients were subsequently able to receive additional therapies. Conclusion: Our results show that this therapeutic doublet represents a clinically effective, safe and welltolerated treatment option for patients with metastatic breast cancer and liver dysfunction.
\end{abstract}

From the early 1970s, cisplatin has been extensively investigated and utilized in the treatment of metastatic breast cancer $(\mathrm{mBC})$ in various lines of therapy, alone and in combination with other chemotherapeutic agents such as vinorelbine, taxanes, anthracyclines or 5-fluorouracil (5-FU).

Correspondence to: Dr. Lorenzo Rossi, Sandro Pitigliani Medical Oncology Department, Hospital of Prato, Via Suor Niccolina Infermiera 20, 59100 Prato, Italy. Tel: +39 0574802520, Fax: +39 0574802903,e-mail: lorenzo.rossi@uslcentro.toscana.it

Key Words: Metastatic breast cancer, liver metastases, cisplatin, 5-fluorouracil, salvage chemotherapy.
In previously untreated patients with $\mathrm{mBC}$, cisplatin and carboplatin led to a response rate (RR) of $50 \%$ and $32 \%$, respectively $(1,2)$. In first-line treatment of $\mathrm{mBC}$, high activity has been observed using combination of platinumbased agents with taxanes or vinorelbine (RRs $\sim 60 \%)(1,3-$ 5). However, the use of platinum salts in the treatment of $\mathrm{mBC}$ did not really develop until a few years ago. Interest in platinum agents has recently been renewed following results from preclinical studies showing synergy of platinum salts with the monoclonal antibody trastuzumab in breast cancer cells with overexpression of erb-b2 receptor tyrosine kinase 2 (human epidermal growth factor receptor 2; HER2) $(6,7)$. More recently, platinum has been suggested as a promising agent in the treatment of triple-negative breast cancer (TNBC) $(8,9)$.

5-FU is not commonly used as a single agent in the treatment of early or metastatic BC, with the exception of capecitabine (an oral prodrug of 5-FU), which is utilized in the metastatic setting, either as monotherapy (10) or in combination with agents such as lapatanib (11). In contrast, intravenous 5-FU is often incorporated into specific chemotherapy protocols such as FAC (5-FU, doxorubicin and cyclophosphamide), FEC (5-FU, epirubicin and cyclophospha-mide), and CMF (cyclophosphamide, methotrexate and 5-FU). Indeed, a recent study by Gruppo Italiano Mammella (12) reappraised the role of 5-FU in these combination therapies, demonstrating that addition of 5-FU to a sequential anthracycline-cyclophosphamide and taxane regimen was not associated with an improved disease-free survival outcome. The combination of platinum salts with 5-FU, widely used in the treatment of other neoplasms, has been poorly studied in $\mathrm{mBC}$, and the data available in the literature are not conclusive (13-16).

The management of $\mathrm{mBC}$ is complex, with overall goals to improve survival, delay disease progression, prolong duration of response, palliate symptoms and to improve or maintain 
quality of life. In order to balance the positive effect of treatment with its associated toxicity, the most important international guidelines suggest the use of sequential regimens of chemotherapy rather than polychemotherapy in settings in which endocrine therapy cannot be used $(17,18)$. However, recommendations are often escalated in the case of lifethreatening, visceral crises or highly symptomatic progression of disease. In the latter context, guidelines suggest combination regimens commonly used in the adjuvant setting (i.e. FAC, FEC, AC, EC, CMF) or others (gemcitabine/paclitaxel; docetaxel/capecitabine; gemcitabine/carboplatin). In the clinical setting, it is not uncommon to encounter scenarios wherein the use of anthracyclines, vinca alkaloids or taxanes is contraindicated due to associated toxicities, or poor end-organ function. This is especially so in patients with impaired liver function (e.g. secondary to hepatic metastases, comorbid liver disease, or due to previous treatment toxicities) who may present with visceral crises requiring urgent chemotherapy. Platinum salts and 5-FU have the comparative advantage of not being extensively metabolized in the liver, and therefore can be safely used in the case of severe liver impairment.

We conducted a retrospective observational study in order to evaluate the efficacy and safety of the combination of platinum salts and 5-FU (PF) in patients with $\mathrm{mBC}$, with particular attention being paid to the subgroup of patients for whom other chemotherapeutic agents were avoided due to impaired hepatic dysfunction reflected by cholestatic or liver function indices.

\section{Patients and Methods}

This descriptive retrospective study reports data extracted from the database of the breast unit of Sandro Pitigliani Department of Medical Oncology, Hospital of Prato, Italy. After identifying patients treated with PF from November 2003 to July 2017, medical records were reviewed and included in this analysis.

The PF protocol consisted of intravenous cisplatin $\left(25 \mathrm{mg} / \mathrm{m}^{2} /\right.$ day $)$ on day $1-3$ and $5-\mathrm{FU}\left(750 \mathrm{mg} / \mathrm{m}^{2} /\right.$ day $)$ given as a continuous intravenous infusion on days 1-3. Cycles were repeated every 21 days until disease progression or unacceptable toxicities. Replacement of cisplatin by carboplatin (area under the curve, AUC $=4$ ) was allowed in the setting of pre-existing contraindication, intolerance, or renal failure (creatinine $\geq 2$ times the baseline level, corresponding to grade 2 or more according to Common Terminology Criteria for Adverse Events (CTCAE) criteria version 4.02) (19). All patients who received other cytotoxic drugs concomitant with PF were excluded from the analysis. Patients who received targeted therapy in combination with PF were included. Patients were followed-up until death or until their most recent recorded visit up to January 2018.

Time to progression (TTP) was calculated from the day of commencement of PF treatment until the day of documented progression (radiological or clinical). Overall survival (OS) was calculated from the start date of PF therapy to the date of death from any cause; patients lost to follow-up were censored in OS calculation at the point of loss. Treatment-related toxicity was retrospectively evaluated according to CTCAE criteria version 4.02 (19).
In the subset of patients with liver metastases, the effect of PF on liver function indices was evaluated: alanine transaminase (ALT), aspartate transaminase (AST), gamma glutamyltransferase (GGT) and bilirubin at the end of treatment were compared to baseline levels recorded before treatment initiation. The distribution of patients at the beginning and at the end of the treatment according to the different CTCAE (v 4.02) grades of hepatic dysfunction for each parameter was evaluated. For each patient, the degree of alteration of liver function hepatic indices before and after PF therapy was evaluated. The change from one CTCAE grade to another was considered an indicator of improvement or worsening of liver function, taking into consideration the parameter with the worst trend.

Alkaline phosphatase (ALP) was excluded from evaluation due to the potential confounding factor caused by the presence of coexisting bone metastases. Descriptive statistics were used to analyze the results.

\section{Results}

Patient characteristics are shown in Table I. During the audited time period, 109 patients with $\mathrm{mBC}$ were treated with PF; at diagnosis, 85 patients $(78 \%)$ had ductal invasive carcinoma versus seven (6.4\%) with lobular invasive carcinoma. Most of the population (56\%) exhibited a biological profile generally considered more aggressive (luminal B-like, luminal B-like/HER2-enriched, HER2positive, or TNBC according to the St Gallen criteria, 2015 (20). In $32.1 \%$ of cases, it was not possible to classify disease according to St Gallen criteria for lack of data related to one of the biological parameters of disease.

Seventy-two patients $(66 \%)$ had liver metastases; five patients $(4.6 \%)$ had de novo metastatic disease. The majority of the patients were heavily pre-treated: $14.7 \%$ received PF as first-line chemotherapy for metastatic disease, $18.3 \%$ as second-line, $11 \%$ as third-line and $55.9 \%$ as fourth-line or beyond. The median number of previous cyototoxic lines of therapy before PF was four (range: 1-10). In metastatic setting, 20 patients $(18.3 \%)$ had previously received anthracyclines, 39 (35.8\%) taxanes, 81 (74.3\%) vinka alkaloids and $36(33 \%)$ capecitabine. Almost all patients (95\%) had previously received anthracyclines or taxanes in the adjuvant setting. Pretreatment with a platinum salt was reported in one patient (cisplatin) in the neoadjuvant setting, and in seven patients (carboplatin) in the metastatic setting. Sixty percent of the patients had been exposed to prior endocrine therapy in the metastatic setting (including progestins for palliative purposes), $52.3 \%$ to prior targeted therapy such as bevacizumab (8.8\%), anti-HER2 agents $(30.7 \%)$ or other agents $(12.8 \%)$. Twenty-one patients (19.3\%) received trastuzumab concomitant with PF.

In the calculation of TTP, 10 patients were excluded from analysis due to lack of data, therefore the calculation of TTP was performed on 99 patients. Twenty patients were lost to follow-up after progression; they were censored in the calculation of the OS. Four additional patients were omitted 
Table I. Patient characteristics and prior treatments $(n=109)$.

\begin{tabular}{|c|c|}
\hline Characteristic & Value \\
\hline \multicolumn{2}{|l|}{ All patients } \\
\hline \multicolumn{2}{|l|}{ Age (years) } \\
\hline Median (range) & $55(30-82)$ \\
\hline \multicolumn{2}{|l|}{ ECOG PS (at cycle 1 of PF), $n(\%)$} \\
\hline 0 & 29 (26.6) \\
\hline 1 & $46(42.2)$ \\
\hline 2 & $21(19.3)$ \\
\hline 3 & $12(11)$ \\
\hline 4 & $1(0.9)$ \\
\hline \multicolumn{2}{|l|}{ Histology, n (\%) } \\
\hline Ductal invasive carcinoma & $85(78)$ \\
\hline Lobular invasive carcinoma & $7(6.4)$ \\
\hline Mixed ductal and lobular invasive carcinoma & $10(9.2)$ \\
\hline Other or not specified & $7(6.4)$ \\
\hline \multicolumn{2}{|l|}{ Immunohistochemical subgroup* } \\
\hline Luminal A & $13(12)$ \\
\hline Luminal B & $33(30.3)$ \\
\hline Luminal B HER2-enriched & $5(4.6)$ \\
\hline HER2-positive & $13(11.9)$ \\
\hline Triple-negative & $10(9.2)$ \\
\hline Not available & $35(32.1)$ \\
\hline \multicolumn{2}{|l|}{ No. of disease site(s) } \\
\hline 1 & $22(20.2)$ \\
\hline 2 & $36(33)$ \\
\hline 3 & $31(28.4)$ \\
\hline$\geq 4$ & $20(18.3)$ \\
\hline \multicolumn{2}{|l|}{ Patients with liver metastases } \\
\hline $\mathrm{n}(\%)$ & $72(66)$ \\
\hline \multicolumn{2}{|l|}{ Patients with metastases $a b$ initio } \\
\hline $\mathrm{n}(\%)$ & $5(4.6)$ \\
\hline \multicolumn{2}{|l|}{ No. of prior lines of chemotherapy for $\mathrm{mBC}, \mathrm{n}(\%)$} \\
\hline 0 (PF as first-line) & $16(14.7)$ \\
\hline 1 & $20(18.3)$ \\
\hline 2 & $12(11)$ \\
\hline 3 & $20(18.3)$ \\
\hline$\geq 4$ & $41(37.6)$ \\
\hline \multicolumn{2}{|l|}{ Previous exposure for $\mathrm{mBC}, \mathrm{n}(\%)$} \\
\hline Anthracyclines & $20(18.3)$ \\
\hline Taxanes & $39(35.8)$ \\
\hline Vinca alkaloids & $81(74.3)$ \\
\hline Capecitabine & $36(33)$ \\
\hline Other & $33(30.3)$ \\
\hline \multicolumn{2}{|l|}{ Concomitant treatment with targeted therapy, $\mathrm{n}(\%)$} \\
\hline Anti-HER2 agent & $21(19.3)$ \\
\hline Other targeted therapy & $0(0)$ \\
\hline Prior treatment with platinum & $8(7.3)$ \\
\hline
\end{tabular}

ECOG PS: Eastern Cooperative Oncology Group performance status; PF: platinum salts and fluorouracil; HER2: human epidermal growth factor receptor 2. *At diagnosis, according to St. Gallen 2015 (20).

due to lack of data. Overall, 105 patients were evaluated for OS. The median TTP was 3.4 months (range $=0-33$ months) (Figure 1). The median OS was 7.8 months (range $=0-122$ months) (Figure 2).
Considering the efficacy results according to treatment line, median TTP was 5.4 months (range $=1-33$ months) in patients who received PF as first -or second-line treatment, and 3.1 months (range $=0-30$ months) in patients who received $\mathrm{PF}$ as a third- or subsequent line. Similarly, the median OS was 8.5 months (range $=1-122$ months) and 6 months (range $=0-70$ months), respectively, in favor of those who received PF in the first-or second-line of treatment.

The progression of liver dysfunction in most patients with liver metastases (93\%) was analyzed. Due to the retrospective nature of the study, in five cases (6.9\%), these data were unavailable. The frequency of patients according to liver-biochemical alterations are shown in Table II, both at the start and at the end of the treatment.

Considering the degree of change in serum liver function values as a surrogate marker of improvement or deterioration of liver function, a complete normalization of liver parameters (both cytolytic and cholestatic) was observed in 20 cases (29.9\%), an improvement in six cases (9\%), worsening in 14 cases $(20.9 \%)$, stable function in eight cases $(12 \%)$ and a biochemically mixed response in two cases (3\%). Seventeen patients $(25.4 \%)$ maintained normal hepatic parameters throughout PF treatment. Overall, 34 patients (50.7\%) obtained a complete, partial or stable biochemical response (Table III).

After PF treatment, 24 patients were able to receive additional cytotoxic drug lines. Four received anthracyclines, 14 taxanes, 12 vinca alkaloids, nine received capecitabine and 14 received other categories of chemotherapeutic drugs.

Treatment with PF was mainly characterized by gastrointestinal toxicity (nausea, vomiting), asthenia and mucositis, occasional myelotoxicity with leukopenia and thrombocytopenia, in most cases of grade 2 (Table IV). Grade 3 thrombocytopenia was observed in two cases, and two cases of febrile neutropenia were reported. In one case, cisplatin was replaced with carboplatin due to grade 2 renal dysfunction. No grade 4 non-hematological toxicities were recorded.

\section{Discussion}

Among the many cytotoxic drugs used in the treatment of $\mathrm{mBC}$, anthracyclines, taxanes, capecitabine and vinca alkaloids are commonly utilized. Platinum-based agents (mainly cisplatin and carboplatin) used as single agent are mildly active in this setting, with some differences in toxicity and activity observed between cisplatin (mean RR 50\%) and carboplatin (mean RR $\sim 30 \%)(1,2)$. Response rates tend to be lower in women who have received prior chemotherapy for metastatic disease $(21,22)$.

Since 1970, several studies have evaluated the impact of cisplatin in BC [reviewed in (1)], but following the advent of anthracyclines and taxanes, platinum salts have been progressively less utilized. Currently, interest in platinum 


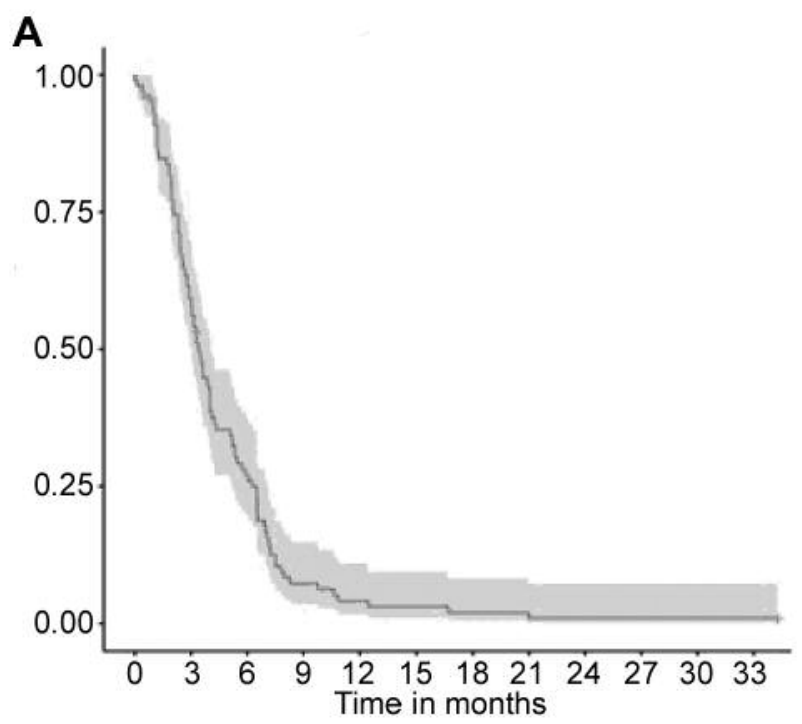

B

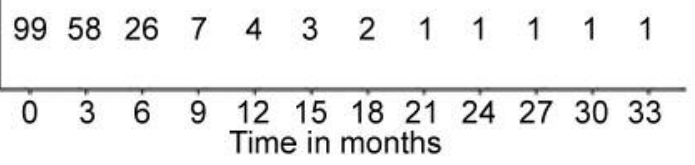

Figure 1. Time to progression. A: Progression probability curve, B: Number of patients at risk of progression of disease. Ten observations were deleted due to missing data.

agents is largely centered on the treatment of TNBC, mainly due to the pharmacodynamic mechanism of the drug $(23,24)$. Some TNBCs exhibit intrinsic genomic instability caused by deficient DNA repair, which facilitates the success of platinum agents in treatment (25), leading to the introduction of schedules containing platinum into the adjuvant or neoadjuvant setting. In metastatic disease, however, the current data are weaker and conflicting. Some studies have shown positive activity of platinum salts in $\mathrm{mBC}(2,26-29)$.

The Translational Breast Cancer Research Consortium 001 study showed a RR of $18 \%$ with the combination of carboplatin $(\mathrm{AUC}=2)$ and cetuximab in 100 patients with TNBC (8). Conversely, the Triple-Negative Breast Cancer Trial, a large phase III trial that randomized approximately 400 patients with metastatic TNBC to receive either single-agent carboplatin or docetaxel, showed no superior activity in favor of carboplatin over docetaxel in unselected patients. However, in patients with germline mutations of breast cancer susceptibility genes (BRCA), a benefit for carboplatin was observed [median progression-free-survival $(\mathrm{PFS})=6.8 \quad(95 \% \quad \mathrm{CI}=4.4$ to 8.1$)$ months versus 3.1 (95\% $\mathrm{CI}=2.4$ to 4.2$)$ months in patients without BRCA mutation] (9). A recent Cochrane meta-analysis evaluated 24 randomized controlled trials, involving 4,418 women, regardless of their immunohistochemical subgroup
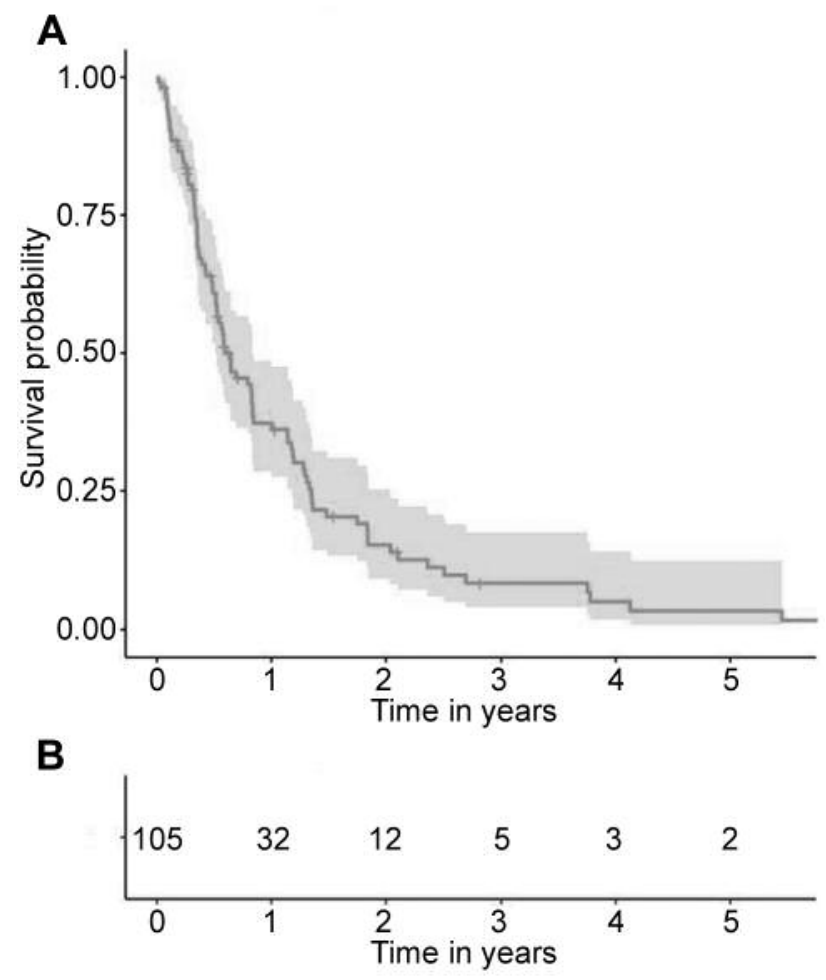

Figure 2. Overall survival. A: Survival probability curve, B: Number of patients at risk of death. Four observations were deleted due to missing data.

(30). The authors concluded that in women with non-metastatic TNBC there was little or no survival benefit in terms of PFS or TTP, at the cost of relevant toxicity (grade 3 or 4 ) both in women treated with cisplatin (nausea/vomiting $\mathrm{RR}=2.65,95 \%$ $\mathrm{CI}=2.10$ to 3.34 , 1,731 women; anemia grade: $\mathrm{RR}=3.72,95 \%$ $\mathrm{CI}=2.36$ to $5.88,1,644$ women) and those treated with carboplatin (nausea/vomiting: $\mathrm{RR}=0.77,95 \% \mathrm{CI}=0.47$ to 1.26 , 1,441 women; $\mathrm{RR}=1.72,95 \% \mathrm{CI}=1.10$ to $2.70,1,441$ women). Overall there was also grade 3 or 4 leukopenia $(\mathrm{RR}=1.38,95 \%$ $\mathrm{CI}=1.21$ to $1.57,3,176$ women) and grade 1 or 2 hair loss ( $\mathrm{RR}=1.41,95 \% \mathrm{CI}=1.26$ to $1.58,1,452$ women).

Fluorouracil is not a drug conventionally used as monotherapy for the treatment of BC, however, 5-FU boluses are mainly used in association with other agents in combined protocols such as CMF, FAC, FEC, etc. (12). In the literature, there is a paucity of data regarding the combination of platinum-based agents (cisplatin or carboplatin) and 5-FU in $\mathrm{mBC}$ (13-16), and there are no data specifically addressing efficacy and safety in patients with liver metastases and visceral crisis requiring salvage chemotherapy.

In this retrospective study, we evaluated 109 women with $\mathrm{mBC}$, regardless of their immunohistochemical panel. The aim of the study was to evaluate the efficacy and safety of the PF association in this setting, especially in those women 
Table II. Progression of liver function tests only in patients with liver metastases (n=67) according to Common Terminology Criteria for Adverse Events criteria (v. 4.02) (19).

\begin{tabular}{|c|c|c|}
\hline Parameter & $\begin{array}{c}\text { At the beginning } \\
\text { of PF, } \mathrm{n}(\%)\end{array}$ & $\begin{array}{l}\text { At the end } \\
\text { of PF, n (\%) }\end{array}$ \\
\hline \multicolumn{3}{|l|}{ AST (highest) } \\
\hline Up to ULN & $26(38.8)$ & $41(61.2)$ \\
\hline$>\mathrm{ULN}-2.5 \times \mathrm{ULN}$ & $17(25.4)$ & $13(19.4)$ \\
\hline$>2.5-5.0 \times \mathrm{ULN}$ & $17(25.4)$ & $4(6)$ \\
\hline$>5.0-20.0 \times \mathrm{ULN}$ & $6(9)$ & $8(11.9)$ \\
\hline$>20.0 \times \mathrm{ULN}$ & $1(1.5)$ & $0(0)$ \\
\hline Not evaluable or missing data & $0(0)$ & $1(1.5)$ \\
\hline \multicolumn{3}{|l|}{ ALT } \\
\hline Up to ULN & $24(35.8)$ & $39(58.2)$ \\
\hline$>\mathrm{ULN}-2.5 \times \mathrm{ULN}$ & $26(38.8)$ & $18(26.9)$ \\
\hline$>2.5-5.0 \times \mathrm{ULN}$ & $14(20.9)$ & $7(10.4)$ \\
\hline$>5.0-20.0 \times \mathrm{ULN}$ & $2(3)$ & $3(4.5)$ \\
\hline$>20.0 \times U L N$ & $1(1.5)$ & $0(0)$ \\
\hline Not evaluable or missing data & $0(0)$ & $0(0)$ \\
\hline \multicolumn{3}{|l|}{ GGT } \\
\hline Up to ULN & $28(41.8)$ & $37(55.2)$ \\
\hline$>\mathrm{ULN}-2.5 \times \mathrm{ULN}$ & $6(9)$ & $4(6)$ \\
\hline$>2.5-5.0 \times \mathrm{ULN}$ & $10(14.9)$ & $8(11.9)$ \\
\hline$>5.0-20.0 \times \mathrm{ULN}$ & $11(16.4)$ & $6(9)$ \\
\hline$>20.0 \times \mathrm{ULN}$ & $6(9)$ & $7(10.4)$ \\
\hline Not evaluable or missing data & $6(9)$ & $5(7.5)$ \\
\hline \multicolumn{3}{|l|}{ Bilirubin } \\
\hline Up to ULN & $41(61.2)$ & $50(74.6)$ \\
\hline$>\mathrm{ULN}-1.5 \times \mathrm{ULN}$ & $15(23.4)$ & $8(11.9)$ \\
\hline$>1.5-3.0 \times$ ULN & $8(11.9)$ & $2(3)$ \\
\hline$>3.0-10.0 \times \mathrm{ULN}$ & $3(4.5)$ & $7(10.4)$ \\
\hline$>10.0 \times \mathrm{ULN}$ & $0(0)$ & $0(0)$ \\
\hline Not evaluable or missing data & $0(0)$ & $0(0)$ \\
\hline
\end{tabular}

AST: Aspartate aminotransferase; ALT: alanine aminotransferase; GGT: gamma-glutamyltransferase; ULN: upper limit of normal; PF: platinum salts and fluorouracil.
Table III. Outcomes of liver function tests $(n=67)$ according to Common Terminology Criteria for Adverse Events (CTCAE) criteria (v. 4.02) (19) in patients with liver metastases $(67 / 72,93 \%)$.

\begin{tabular}{lc}
\hline Outcome & $\mathrm{N}(\%)$ \\
\hline Maintenance of normal liver parameters & $17(25.4)$ \\
Complete normalization of liver parameters & $20(29.9)$ \\
Improvement in liver function* & $6(9)$ \\
Worsening of liver function* & $14(20.9)$ \\
Stability of liver function** & $8(12)$ \\
Biochemical mixed response & $2(3)$ \\
\hline
\end{tabular}

* Considered a transition from one CTCAE class of alteration of aspartate aminotransferase (AST) alanine aminotransferase (ALT), gamma-glutamyltransferase (GGT) or bilirubin (the one with the worst trend) to a lower class. ${ }^{* *}$ Considered maintenance within the same CTCAE class of alteration of alkaline phosphatase, ALT, GGT or bilirubin (the one with the worst trend).

Table IV. Platinum and fluorouracil treatment-related adverse events according to Common Terminology Criteria for Adverse Events criteria (v. 4.02) (19)

\begin{tabular}{lcc}
\hline Event & Grade $1 / 2, \mathrm{n}(\%)$ & Grade 3/4, n (\%) \\
\hline Myelotoxicity & $20(18.3)$ & $4(3.7)$ \\
Peripheral neuropathy & $5(4.6)$ & $0(0)$ \\
Nausea/vomiting & $32(29.4)$ & $2(1.8)$ \\
Stomatitis & $5(4.5)$ & $1(0.9)$ \\
Acute renal impairment & $4(3.7)$ & $0(0)$ \\
Fatigue & $11(10.1)$ & $0(0)$ \\
Constipation & $2(1.8)$ & $0(0)$ \\
Diarrhea & $6(5.5)$ & $0(0)$ \\
Tinnitus & $1(0.9)$ & $0(0)$ \\
Anorexia & $4(3.7)$ & $0(0)$ \\
\hline
\end{tabular}

predominantly of patients with significant metastatic burden, aggressive disease, and with a history of heavy pretreatment who were receiving PF as salvage therapy.

Nevertheless, it is notable that there were seven patients who demonstrated a long TTP (range=12 to 33 months) and excellent OS (up to 122 months). Six of these patients experienced significant improvements in liver function tests after receiving PF, and subsequently received active drugs that would otherwise have been contraindicated due to hepatic dysfunction, such as taxanes, anthracyclines or eribulin.

The overall goals of treatment in patients with $\mathrm{mBC}$ are mainly prolongation of survival and alleviation of symptoms, therefore, the choice of therapeutic agents must be carefully made with attention to their toxicity profiles. The main adverse effects of PF are nausea, vomiting and peripheral neuropathy. In the present case series, these side-effects were mild or moderate (Table II), and overall, the PF regimen was 
generally well-tolerated and safe, raising no concerns in terms of hepatic toxicities. Similarly to what has been observed in the treatment of other tumor types $(31,32)$, we believe that the mode of delivery of PF, characterized by a 3-day split schedule for both agents with 5-FU in continuous infusion, rather than in bolus, may have reduced the risk of serious toxicity and improved compliance.

To our knowledge, this is the only study evaluating the efficacy and safety of PF in a sizeable group of patients with $\mathrm{mBC}(>100)$; specifically focusing on patients with hepatic metastatic disease. In this study, the administration of PF resulted in the improvement of liver function in many patients, with $29.9 \%$ of cases experiencing a complete normalization of all the studied hepatic indices. All cases of observed worsening of hepatic function occurred concurrently with progression liver disease, therefore we believe these worsening parameters were not primarily attributable to PFrelated hepatotoxicity. Indeed, our results suggest that PF may have potential for use in earlier treatment lines in patients with deranged liver biochemistry, in order to reverse this situation and allow for use of other cytotoxic agents in subsequent lines that would otherwise be contraindicated due to hepatic dysfunction. Our findings are limited due to the fact they were derived retrospectively from a single institution, but nevertheless warrant further consideration by other groups.

On the basis of these results, the combination of a platinum agent and 5-FU in continuous infusion still represents a treatment option in $\mathrm{mBC}$. This option may be of particular interest for patients with liver metastases and associated derangement of liver function tests who require a salvage therapy with a low hepatic toxicity profile. Reduction of live function test indices may allow patients to receive potentially hepatotoxic drugs in subsequent lines of therapies. Further studies in larger case series will be able to clarify the role of this combination therapy in this particular frail subgroup of patients.

\section{Conflicts of Interest}

All the Authors declare there are no conflicts of interest in regard to this study.

\section{Ethical Approval}

Ethical consent was not required due to the nature of the study (retrospective analysis of recorded data).

\section{References}

1 Decatris MP, Sundar S and O'Byrne KJ: Platinum-based chemotherapy in metastatic breast cancer: current status. Cancer Treat Rev 30(1): 53-81, 2004.

2 Martin M, Diaz-Rubio E, Casado A, Santabárbara P, López Vega JM, Adrover E and Lenaz L. Carboplatin: an active drug in metastatic breast cancer. J Clin Oncol 10: 433-437, 1992.
3 Mustacchi G, Muggia M, Milani S, Ceccherini R, Leita ML and Dellach C: A Phase II study of cisplatin and vinorelbine in patients with metastatic breast cancer. Ann Oncol 12(11): 17301736, 2002.

4 Li M, Fan Y, Li Q, Zhang P, Yuan P, Ma F, Wang J, Luo Y, Cai $\mathrm{R}$, Chen S, Li Q and Xu B: Vinorelbine Plus Platinum in Patients with Metastatic Triple Negative Breast Cancer and Prior Anthracycline and Taxane Treatment. Medicine (Baltimore) 94(43): 1928-1934, 2015.

5 Vassilomanolakis M, Koumakis G, Barbounis V, Demiri M, Panopoulos C, Chrissohoou M, Apostolikas N and Efremidis AP: First-line chemotherapy with docetaxel in metastatic breast cancer. Breast 14(2): 136-141, 2005.

6 Huang R, Sun Y, Gao Q, Wang Q and Sun B: Trastuzumabmediated selective delivery for platinum drug to HER2-positive breast cancer cells. Anticancer Drugs 26(9): 957-963, 2015.

7 Ding X, Qu X, Fan Y, Che X, Qu J, Xu L, Liu J and Liu Y: Trastuzumab and oxaliplatin exhibit a synergistic antitumor effect in HER2-postive gastric cancer cells. Anticancer Drugs 25(3): 315-322, 2014.

8 Carey LA, Rugo HS, Marcom PK, Mayer EL, Esteva FJ, Ma CX, Liu MC, Storniolo AM, Rimawi MF, Forero-Torres A, Wolff AC, Hobday TJ, Ivanova A, Chiu WK, Ferraro M, Burrows E, Bernard PS, Hoadley KA, Perou CM and Winer EP: TBCRC 001: EGFR inhibition with cetuximab added to carboplatin in metastatic triple-negative (basal-like) breast cancer. J Clin Oncol 26(15S): 1009 (supplement), 2008.

9 Tutt A, Ellis P, Kilburn L, Gillett C, Pinder S, Abraham J, Barrett S, Barrett-Lee P, Chan S, Cheang M, Dowsett M, Fox L, Gazinska P, Grigoriadis A, Gutin A, Harper-Wynne C, Hatton M, Kernaghan S, Lanchbury J, Morden J, Owen J, Parikh J, Parker P, Rahman N, Roylance R, Shaw A, Smith I, Thompson R, Timms K, Tovey H, Wardley A, Wilson G, Harries M and Bliss J: TNT: A randomized phase III trial of carboplatin compared with docetaxel for patients with metastatic or recurrent locally advanced triple negative or BRCA1/2 breast cancer. Presented at: San Antonio Breast Cancer Symposium; 2014.

10 Blum JL, Jones SE, Buzdar AU, LoRusso PM, Kuter I, Vogel C, Osterwalder B, Burger HU, Brown CS and Griffin T: Multicenter phase II study of capecitabine in paclitaxel-refractor metastatic breast cancer. J Clin Oncol 17: 485-493, 1999.

11 Geyer CE, Forster J, Lindquist D, Chan S, Romieu CG, Pienkowski T, Jagiello-Gruszfeld A, Crown J, Chan A, Kaufman B, Skarlos D, Campone M, Davidson N, Berger M, Oliva C, Rubin SD, Stein S and Cameron D: Lapatanib plus capecitabine for HER2-positive advanced breast cancer. N Engl J Med 355: 2733-2743, 2006.

12 Del Mastro L, De Placido S, Bruzzi P, De Laurentiis M, Boni C, Cavazzini G, Durando A, Turletti A, Nisticò C, Valle E, Garrone O, Puglisi F, Montemurro F, Barni S, Ardizzoni A, Gamucci T, Colantuoni G, Giuliano M, Gravina A, Papaldo P, Bighin C, Bisagni G, Forestieri V, Cognetti $F$ and Gruppo Italiano Mammella (GIM) investigators: Fluorouracil and dose-dense chemotherapy in adjuvant treatment of patients with early-stage breast cancer: an open-label, $2 \times 2$ factorial, randomised phase 3 trial. Lancet 385(9980): 1863-1872, 2015.

13 Sun S, Wang LP, Zhang J, Yang XY, Zhang QL, Jia Z, Hu XC and Wang BY: Phase II study of oxaliplatin plus leucovorin and 5 -fluorouracil in heavily pretreated metastatic breast cancer patients. Med Oncol 29: 418-424, 2012. 
14 Pectasides D, Pectasides M, Farmakis D, Gaglia A, Koumarianou A, Nikolaou M, Koumpou M, Kountourakis P, Papaxoinis G, Mitrou P, Economopoulos T and Raptis SA: Oxaliplatin plus high-dose leucovorin and 5-fluorouracil in pretreated advanced breast cancer: a phase II study. Ann Oncol 14: 537-542, 2003.

15 Zelek L, Cottu P, Tubiana-Hulin M, Vannetzel JM, Chollet P, Misset JL, Chouaki N, Marty M, Gamelin E, Culine S, Dieras $\mathrm{V}$, Mackenzie S and Spielmann M: Phase II study of oxaliplatin and fluorouracil in taxane and anthracycline-pretreated breast cancer patients. J Clin Oncol 20: 2551-2558, 2002.

16 Lekakis L, Tryfonopoulos D, Pistamatzian N, Panopoulos C, Koumakis G, Demiri S and Efremidis A: Salvage chemotherapy with cisplatin and 5-fluorouracil in metastatic breast cancer. Particular activity against liver metastases. Anticancer Res 32(5): 1833-1837, 2012.

17 Cardoso F, Costa A, Senkus E, Aapro M, André F, Barrios CH, Bergh J, Bhattacharyya G, Biganzoli L, Cardoso MJ, Carey L, Corneliussen-James D, Curigliano G, Dieras V, El Saghir N, Eniu A, Fallowfield L, Fenech D, Francis P, Gelmon K, Gennari A, Harbeck N, Hudis C, Kaufman B, Krop I, Mayer M, Meijer H, Mertz S, Ohno S, Pagani O, Papadopoulos E, Peccatori F, Penault-Llorca F, Piccart MJ, Pierga JY, Rugo H, Shockney L, Sledge G, Swain S, Thomssen C, Tutt A, Vorobiof D, Xu B, Norton L and Winer E: 3rd ESO-ESMO International Consensus Guidelines for Advanced Breast Cancer (ABC 3). Ann Oncol 28(1): 16-33, 2017.

18 NCCN Clinical Practice Guidelines in Oncology (NCCN Guidelines) Breast Cancer. Version 4.2017 - February 7, 2018.

19 National Cancer Institute. Common Terminology Criteria for Adverse Events v4.02. NCI, NIH, DHHS. May 29, 2009. NIH publication \# 09-7473.

20 Coates AS, Winer EP, Goldhirsch A, Gelber RD, Gnant M, Piccart-Gebhart M, Thürlimann B and Senn HJ: Tailoring therapies-improving the management of early breast cancer: St Gallen International Expert Consensus on the Primary Therapy of Early Breast Cancer 2015. Ann Oncol 26: 1533-1546, 2015.

21 Kolaric K and Roth A: Phase II clinical trial of cisdichlorodiammine platinum (cis-DDP) for antitumorigenic activity in previously untreated patients with metastatic breast cancer. Cancer Chemother Pharmacol 11(2): 108-112, 1983.

22 Sledge GW Jr., Loehrer PJ Sr., Roth BJ and Einhorn LH: Cisplatin as first-line therapy for metastatic breast cancer. J Clin Oncol 6(12): 1811-1814, 1988.
23 Leong CO, Vidnovic N, Deyoung MP, Sgroi D and Ellisen LW: The p63/p73 network mediates chemosensitivity to cisplatin in a biologically defined subset of primary breast cancers. J Clin Invest 117(5): 1370-1380, 2007.

24 Kelland L: The resurgence of platinum-based cancer chemotherapy. Nat Rev Cancer 7: 573-584, 2007.

25 Agrawal LS and Mayer IA: Platinum agents in the treatment of early-stage triple-negative breast cancer: is it time to change practice? Clin Adv Hematol Oncol 12(10): 654-658, 2014.

26 Crown JP: The platinum agents: a role in breast cancer treatment? Semin Oncol 28(1 Suppl 3): 28-37, 2001.

27 Kolaric K and Vukas D: Carboplatin activity in untreated metastatic breast cancer patients - results of a phase II study. Cancer Chemother Pharmacol 27: 409-412, 1991.

28 Carmo-Pereira J, Oliveira Costa F, Henriques E and Rubens RD: Carboplatin (C) as primary chemotherapy for disseminated breast carcinoma: a phase II study (abstr.). ECCO-5: P-0971, 1989.

29 O'Brien ME, Talbot DC and Smith IE: Carboplatin in the treatment of advanced breast cancer: a phase II study using a pharmacokinetically guided dose schedule. J Clin Oncol 11: 2112-2117, 1993.

30 Egger SJ, Willson ML, Morgan J, Walker HS, Carrick S, Ghersi $\mathrm{D}$ and Wilcken N: Platinum-containing regimens for metastatic breast cancer. Cochrane Database Syst Rev 23(6): CD003374, 2017.

31 Hansen RM, Ryan L, Anderson T, Krzywda B, Quebbeman E, Benson A 3rd, Haller DG and Tormey DC: Phase III study of bolus versus infusion fluorouracil with or without cisplatin in advanced colorectal cancer. J Natl Cancer Inst 88: 668-674, 1996.

32 Chung YS, Yamashita Y, Inoue T, Matsuoka T, Nakata B, Onoda N, Maeda K, Sawada T, Kato Y, Shirasaka T and Sowa M: Continuous infusion of 5-fluorouracil and low dose cisplatin infusion for the treatment of advanced and recurrent gastric adenocarcinoma. Cancer 80: 1-7, 1997.

Received June 15, 2018

Revised June 25, 2018

Accepted June 28, 2018 